\title{
Criminologie
}

\section{Les femmes autochtones et la justice pénale}

\section{Mylène Jaccoud}

Volume 25, numéro 1, 1992

Les femmes et le contrôle pénal, questions féministes

URI : https://id.erudit.org/iderudit/017315ar

DOI : https://doi.org/10.7202/017315ar

Aller au sommaire du numéro

Éditeur(s)

Les Presses de l'Université de Montréal

ISSN

0316-0041 (imprimé)

1492-1367 (numérique)

Découvrir la revue

Citer cet article

Jaccoud, M. (1992). Les femmes autochtones et la justice pénale. Criminologie, 25(1), 65-85. https://doi.org/10.7202/017315ar

\section{Résumé de l'article}

The interest in understanding and analyzing the situation of native women within the penal process is recent and seems to be limited by the inordinate attention paid by researchers to the overrepresentation of native women. This article is an account of the findings and analyses made to date in this recent sector of research. It presents an inventory of the principal data concerning the confrontation of native women with the penal process. It proposes a synthesis of the principal analyses of the problems of native women with the system of justice by presenting a critical analysis of the socio-structural model of LaPrairie.
Ce document est protégé par la loi sur le droit d'auteur. L'utilisation des services d'Érudit (y compris la reproduction) est assujettie à sa politique d'utilisation que vous pouvez consulter en ligne.

https://apropos.erudit.org/fr/usagers/politique-dutilisation/ 
The interest in understanding and analyzing the situation of native women within the penal process is recent and seems to be limited by the inordinate attention paid by researchers to the overrepresentation of native women. This article is an ac. count of the findings and analyses made to date in this recent sector of research. It presents an inventory of the principal data concerning the confrontation of native women with the penal process. It proposes a synthesis of the principal analyses of the problems of native women with the system of justice by presenting a critical analysis of the socio-structural model of LaPrairie.

\section{INTRODUCTION}

La situation des femmes autochtones ${ }^{2}$ dans leur relation avec les appareils de contrôle de l'État commence à peine à capter l'attention des chercheur-e-s. Mais les études sont encore clairsemées et restent, à quelques exceptions près, très superficielles, en raison surtout d'un intérêt démesuré pour la surreprésentation des femmes autochtones, surreprésentation à laquelle, est-il besoin de le rappeler, les hommes autochtones n'échappent pas. Cet article propose de faire le bilan des connaissances et des analyses élaborées jusqu'à ce jour dans ce récent secteur de recherche.

\section{LA PLACE DES FEMMES AUTOCHTONES DANS LES RECHERCHES}

L'intérêt criminologique pour les autochtones correspond à la montée d'une conscience nationale aborigène qui se fait jour dès l'annonce par le gouvernement Trudeau en 1969 de sa politique d'abolition des réserves amérindiennes. L'incarcération excessive des autochtones est

1. M. Sc., Ecole de criminologie, Universitê de Montréal, case postale 6128, succ. «A », Montréal (Québec) H3C 3J7.

2. Par autochtones, nous référons aux trois catégories officielles, à savoir les Amérindiens, les Métis et les Inuit. 
pour la première fois mise en évidence vers la fin des années 1970 dans l'étude d'un organisme privé travaillant pour le compte du ministère des Affaires indiennes et du Nord (Laing, 1967). Le rapport Laing faisait alors déjà le constat d'une surreprésentation des femmes autochtones dans la prison provinciale de Prince-Albert en août 1966 (Laing, 1967, p. 49), constat qui sera réitéré par la Commission de réforme du droit du Canada quelques années plus tard pour l'établissement de Pine Grove en Saskatchewan (Schmeiser, 1974). Mais aucune étude systématique n'est encore élaborée sur les femmes autochtones. Il faut attendre les années 1980 pour qu'une plus grande attention leur soit accordée, mais les données restent encore très descriptives (voir par exemple Johnson, 1986), et cet intérêt est loin d'être assuré et constant : le récent rapport final d'un groupe d'étude, pourtant spécifiquement orienté vers l'étude des populations autochtones au sein du régime correctionnel fédéral, n'a consacré qu'un simple paragraphe à la situation des femmes autochtones (Canada, Solliciteur général, 1989, p. 28). Ce sont les associations de femmes autochtones qui se feront les instigatrices d'une réflexion sur la condition des femmes autochtones au sein du système correctionnel en tant que corollaire de leur condition au sein de la société euro-canadienne (voir par exemple l'étude de Dubec, 1982). Et il faut attendre les travaux de LaPrairie $(1984 ; 1987 ; 1989$ a) pour qu'enfin s'amorce une réflexion prometteuse sur les rapports que ces femmes entretiennent avec l'appareil pénal. Soulignons que le récent groupe d'étude sur les femmes sous sentence fédérale (Canada. Groupe d'étude sur les femmes purgeant une peine fédérale, 1990), qui compte quelques représentantes des premières nations, s'est largement préoccupé de la question autochtone et a eu l'originalité, parmi le flot de statistiques, de présenter les points de vue de femmes autochtones sur le système de justice euro-canadien ainsi qu'une analyse du traitement que leur réserve ce système.

\section{LIMITES DES ÉTUDES CRIMINOLOGIQUES RELATIVES AUX AUTOCHTONES}

L' « incarcération excessive » des peuples autochtones dans les institutions d'enfermement au Canada sera le point de départ de l'élaboration d'un nouvel objet de recherche criminologique qui a somme toute du mal à se construire en raison du peu d'empressement des chercheurs à déplacer leur centre d'intérêt de l'univers carcéral. Aussi, les études criminologiques portant sur les peuples autochtones sont-elles encore limitées et le travail de défrichement de ce nouveau champ d'étude en 
est à ses débuts. On constate qu'une priorité est accordée aux recherches empiriques de type quantitatif et descriptif. Les études souffrent d'un éclatement nuisible à la généralisation des données. L'effort de theorisation en est à ses premiers balbutiements. Les recherches se confinent au seul problème de la surreprésentation et stagnent encore dans les marécages du carcéral. Et le désintérêt pour les études comparatives basées sur le sexe ne permet pas encore d'échafauder une analyse solide de la sur-criminalisation des femmes autochtones.

Certaines de ces limites, il faut bien le dire, sont dépendantes de restrictions méthodologiques inhérentes à la production statistique touchant les autochtones. Les statistiques produites par la police ${ }^{3}$ et les tribunaux ne distinguent pas les autochtones des allochtones, et ce vide statistique voile encore le schéma qui pourrait rendre compte des pratiques policières et sentencielles à l'égard des femmes autochtones. Les statistiques correctionnelles sont plus completes, mais leur fiabilité est compromise par les conditions suivantes :

$1^{\circ}$ l'enregistrement de l'origine ethnique à l'admission s'effectue soit par auto-déclaration, certaines personnes d'origine ethnique mixte déclareraient alors une origine anglaise ou française plutôt qu'autochtone, soit par identification visuelle du gardien à qui revient le pouvoir d'inscrire l'autochtone dans l'une des trois catégories suivantes: Amérindienne, Métisse ou Inuit ; il semblerait que les Inuit soient parfois confondus avec des gens d'autres ethnies (Cousineau et al., 1986);

$2^{\circ}$ les statistiques provinciales n'enregistrent pas systématiquement les autochtones: les statistiques pénitenciaires sont donc plus complètes. Par exemple, la province de Québec n'a enregistré l'identité des autochtones dans ses établissements de détention provinciaux qu'à partir de 1977 (Jaccoud, 1986);

$3^{\circ}$ les statistiques provinciales sont non seulement incomplètes, mais elles sont de qualité inégale. Ainsi, les régions de l'Ouest canadien, à plus forte concentration démographique autochtone, disposeraient de données plus complètes que dans les provinces de l'Est, ce qui rend les comparaisons interprovinciales hasardeuses ;

$4^{\circ}$ enfin, les taux d'incarcération et les éléments de comparaison entre autochtones et non-autochtones sont difficiles à établir en raison du manque de fiabilité des données démographiques sur les auto-

3. Le service de police de la Conmunauté urbaine de Montréal a commencé cette année seulement à considérer l'origine autochtone dans ses rapports d'événements. 
chtones. Par exemple, la population des Métis est l'une des nations pour lesquelles les recensements démographiques semblent les moins fiables, alors qu'elle constitue l'une des catégories lors de l'admission dans les institutions correctionnelles.

Ces considérations sont importantes quand il s'agit d'évaluer la surreprésentation des femmes autochtones dans le système pénal. À l'exception de la dernière, ces limites méthodologiques rappellent que la surreprésentation des femmes autochtones est sans doute sous-estimée.

\section{LA SURREPRÉSENTATION}

La surreprésentation des femmes autochtones s'observe aussi bien dans les établissements de détention fédéraux que provinciaux. Alors que la population autochtone constituait 1,5 pour cent de la population totale du Canada en 1986 (Statistique Canada, 1989) les femmes autochtones représentaient 14,2 pour cent des détenues incarcérées au pénitencier pour femmes de Kingston, les hommes autochtones 9,6 pour cent de la population carcérale fédérale au 31 mars 1987 (Canada. Solliciteur général, 1989) et des pourcentages nettement plus élevés dans les établissements provinciaux. Ainsi, en 1989-1990, les femmes autochtones constituaient 29,1 pour cent de toutes les femmes admises dans les établissements provinciaux et territoriaux alors que les hommes autochtones comptaient pour 16,9 pour cent des hommes admis (Statistique Canada, 1991). Il semble que l'admission des femmes autochtones soit en nette progression dans les établissements provinciaux et en diminution dans les établissements fédéraux. Par rapport à 1982-1983, la proportion des femmes autochtones a augmenté de près de 11 pour cent dans les établissements provinciaux et a diminué de 4,2 pour cent au pénitencier de Kingston ${ }^{4}$.

4. En 1982-1983, la proportion des détenues autochtones était de 18,4 pour cent au niveau fédéral et de 18,2 pour cent au niveau provincial (Canada, Solliciteur général, 1989). Mais il est délicat de parler d'une tendance à la baisse. Les statistiques fédérales touchent des petits nombres, de sorte que de petites variations dans ces nombres grossissent rapidement les pourcentages. Par exemplc, au 31 décembre 1984, 26 autochtones étaient incarcérées a Kingston sur un total de 130 admises, soit 20 pour cent de l'ensemble des détenues. La proportion des détenues autochtones avait donc augmenté par rapport à 1982-1983 (Service correctionnel Canada, 1985). Ajoutons qu'un plus grand nombre de femmes autochtones choisissent de purger leur sentence fédérale dans des établissements provinciaux en vertu des ententes d'échange de service, ce qui pourrait expliquer la diminution de la proportion de femmes autochtones à Kingston. 
En réitérant les réserves déjà émises en raison des limites des données statistiques, la répartition des admissions des femmes autochtones dans les établissements de détention à travers le Canada révèle que les admissions se concentrent dans les provinces de l'Ouest, puisque les trois quarts des femmes autochtones ont été admises en Alberta $(38,7 \%)$, en Saskatchewan $(24,7 \%)$ et en Ontario (18,8\%). Même en tenant compte des plus fortes concentrations démographiques autochtones dans les provinces de l'Ouest, la surreprésentation reste encore la norme 5 .

Au Québec, l'incarcération des femmes autochtones semble beaucoup moins systématique que dans les provinces de l'Ouest, mais leur surreprésentation y est aussi constatée. De 1977 à 1985 inclusivement, les Amérindiennes ${ }^{6}$ représentaient 2,3 pour cent du total des admissions des femmes (Jaccoud, 1986) alors que les Amérindiens représentaient 0,61 pour cent de la population québécoise (SAGMAI, 1984). Par ailleurs, les Amérindiennes constituaient plus de 10 pour cent de la population amérindienne admise en détention au cours des neuf années considérées alors que les non-Amérindiennes représentaient 5,4 pour cent des admissions durant la même période. Proportionnellement, les Amérindiennes étaient donc deux fois plus nombreuses que les allochtones (voir tableau 1).

Des données récentes du ministère de la Sécurité publique du Québec permettent de dévoiler la répartition ethnique des femmes autochtones admises en détention au Québec au cours des trois dernières années. Les trois quarts de ces femmes sont Amérindiennes alors que le quart restant sont Inuit (voir tableau 2). Les femmes inuit sont en minorité dans les établissements de détention provinciaux et fédéraux ${ }^{7}$, mais c'est au Québec que leur pourcentage en détention est le plus fort ${ }^{8}$

5. Le recensement de 1981 indique que les autochtones representaient 17,5 pour cent au Yukon, 58 pour cent dans les Territoires du Nord-Ouest, 3 pour cent en Colombie-Britannique, 3,3 pour cent en Alberta, 6,2 pour cent en Saskatchewan, 6,5 pour cent au Manitoba, 1,3 pour cent en Ontario, 0,8 pour cent au Québec, 0,8 pour cent au NouveauBrunswick, 0,9 pour cent en Nouvelle-Écosse, 0,5 pour cent à l'île-du-Prince-Édouard et 0,8 pour cent à Terre-Neuve (Statistique Canada, 1981).

6. La distinction entre nations n'ayant pu être saisie lors de notre étude en raison d'erreurs survenues dans la saisie manuelle des données, seule la catégorie des Amérindiennes avait pu être retenue (Jaccoud, 1986).

7. En 1989, deux femmes inuit purgeaient une sentence fédérale à la prison de Tanguay de Montréal (Faulkner, 1989).

8. Alors que les Inuit représentaient 2,4 pour cent des détenus autochtones dans les pénitenciers fédéraux en 1987, le Québec comptait 30,7 pour cent de détenus inuit dans ses pénitenciers (Canada. Solliciteur général, 1989). 
Tableau 1

Admissions des Amérindiens et des non-Amérindiens selon le sexe, 1977-1985, Québec

\begin{tabular}{|c|c|c|c|c|c|c|c|c|c|c|c|c|}
\hline \multirow[b]{3}{*}{ Année } & \multicolumn{5}{|c|}{ Amérindiens } & \multicolumn{7}{|c|}{ Non-Amérindiens } \\
\hline & \multicolumn{2}{|c|}{$H$} & \multicolumn{2}{|c|}{$F$} & \multicolumn{2}{|c|}{ Total } & \multicolumn{2}{|c|}{$H$} & \multicolumn{2}{|c|}{$F$} & \multicolumn{2}{|c|}{ Total } \\
\hline & $\mathrm{N}$ & $\%$ & $\mathbf{N}$ & $\%$ & $\mathbf{N}$ & $\%$ & $\mathrm{~N}$ & $\%$ & $\mathrm{~N}$ & $\%$ & $\mathrm{~N}$ & $\%$ \\
\hline $\begin{array}{l}1977 \\
1978 \\
1979 \\
1980 \\
1981 \\
1982 \\
1983 \\
1984 \\
1985\end{array}$ & $\begin{array}{l}349 \\
474 \\
381 \\
332 \\
345 \\
284 \\
332 \\
260 \\
386\end{array}$ & $\begin{array}{r}11,1 \\
15,1 \\
12,1 \\
10,6 \\
11,0 \\
9,0 \\
10,6 \\
8,2 \\
12,3\end{array}$ & $\begin{array}{l}32 \\
66 \\
49 \\
27 \\
29 \\
36 \\
44 \\
37 \\
43\end{array}$ & $\begin{array}{r}8,8 \\
18,2 \\
13,5 \\
7,5 \\
8,0 \\
9,9 \\
12,1 \\
10,2 \\
11,8\end{array}$ & $\begin{array}{l}381 \\
540 \\
430 \\
359 \\
374 \\
320 \\
376 \\
297 \\
429\end{array}$ & $\begin{array}{r}10,9 \\
15,4 \\
12,3 \\
10,2 \\
10,7 \\
9,1 \\
10,7 \\
8,5 \\
12,2\end{array}$ & 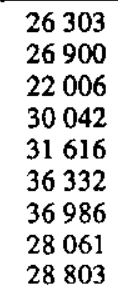 & $\begin{array}{r}9,8 \\
10,1 \\
8,3 \\
11,3 \\
11,8 \\
13,6 \\
13,8 \\
10,5 \\
10,8\end{array}$ & $\begin{array}{r}1490 \\
1550 \\
978 \\
1728 \\
11797 \\
2129 \\
2127 \\
11643 \\
1865\end{array}$ & $\begin{array}{r}9,7 \\
10,1 \\
6,4 \\
11,3 \\
11,7 \\
14,0 \\
13,9 \\
10,7 \\
12,2\end{array}$ & $\begin{array}{l}27793 \\
28450 \\
22984 \\
31770 \\
33413 \\
38461 \\
39113 \\
29704 \\
30668\end{array}$ & $\begin{array}{r}9,8 \\
10,1 \\
8,1 \\
11,3 \\
11,8 \\
13,6 \\
13,9 \\
10,5 \\
10,9\end{array}$ \\
\hline $\begin{array}{l}\text { Total } \\
\text { Valeurs } \\
\text { manquantes }\end{array}$ & 3143 & 100,0 & 363 & 100,0 & 3506 & 100,0 & $\begin{array}{r}267049 \\
-\end{array}$ & 100,0 & 15307 & $\begin{array}{r}100,0 \\
-\end{array}$ & $\begin{array}{r}282.356 \\
-\end{array}$ & 100,0 \\
\hline
\end{tabular}

Source : M. Jaccoud (1986), Les Autochtones dans le système de justice pénale au Québec, thèse de maîtrise, Écolc de criminologie, Université de Montreal, p. 161. 
en raison de leur concentration démographique dans la région du Nouveau-Québec. Les administrateurs et les intervenants du système de justice doivent considérer cette minorité avec attention, car si l'éloignement des Amérindiennes de leur communauté se pose souvent dans le cas d'une détention, le problème est encore plus dramatique pour les Inuit qui ne peuvent regagner leur communauté qu'au terme d'un voyage aérien fort coûteux.

Tableau 2

Admissions des autochtones dans les établissements de détention du Québec selon le sexe et l'année

\begin{tabular}{llrrrr}
\hline & & 1988 & 1989 & \multicolumn{1}{c}{1990} & Total \\
\hline Amérindiens & Hommes & 710 & 810 & 1025 & 2545 \\
\multirow{2}{*}{ Inuit } & Femmes & 85 & 105 & 108 & 298 \\
& Hommes & 134 & 173 & 242 & 549 \\
Total & Femmes & 25 & 32 & 28 & 85 \\
\hline
\end{tabular}

Source : Ministère de la Sécurité publique. Direction générale des Services correctionnels. Gouvernement du Québec. Service évaluation, recherche et planification, juin 1991 .

\section{LA FEMME AUTOCHTONE INCRIMINÉE : POINTS SAILLANTS}

La revue des quelques études ayant traité en tout ou en partie des femmes autochtones permet de faire ressortir certaines caractéristiques majeures de la femme autochtone incriminée :

$1^{\circ}$ Précocité du contact avec l'appareil de justice : des 33 femmes autochtones emprisonnées dans les prisons ontariennes interviewées par Dubec (1982), plus de la moitié avaient déjà été arrêtées entre 14 et 17 ans. Ce constat invite à considérer avec le plus grand soin les mécanismes de contrôle et de sélection s'exerçant à l'endroit des jeunes femmes autochtones, car ils jouent très certainement sur leur trajectoire pénale ultérieure. Malheureusement, les adolescentes autochtones n'ont pas encore fait l'objet de recherches (LaPrairie, 1984).

$2^{\circ}$ Fréquence des démêlés, tant sur le plan des arrestations que sur celui des condamnations. Pour les arrestations, l'étude de Dubec (1982) rapporte que 40 pour cent des femmes autochtones de l'échantillon avaient été arrêtées 15 fois ou plus; l'étude du Centre d'amitié autochtone de Montréal (1986), réalisée auprès de 39 femmes ayant des 
démêlés avec la justice, précise que près des trois quarts n'en étaient pas à leur première arrestation. Quant aux condamnations, 21 pour cent des femmes autochtones de l'échantillon de l'étude de Dubec (1982) avaient au moins 17 incarcérations antérieures. En rappelant la surreprésentation des femmes autochtones dans le système de justice pénale, on peut soutenir que la fréquence des démêlés des femmes autochtones avec le système de justice est doublement importante. Elle est à la fois horizontale et verticale. Une fréquence horizontale compare les démêlés des autochtones avec l'appareil de justice en rapport à ceux des autres femmes et renvoie à la notion de surreprésentation; une fréquence verticale compare la fréquence des démêlés à l'intérieur d'une trajectoire pénale individuelle.

$3^{\circ}$ Importance et incidence des toxicomanies : toutes les études, sans exception, s'accordent pour établir un lien entre la surconsommation de drogues et d'alcool et la fréquence des démêlés avec les agences de contrôle. Le groupe d'étude sur les femmes sous sentence fédérale (Canada,Groupe d'étude sur les femmes purgeant une peine fédérale, 1990) rapporte que l'abus de substances psychoactives était plus élevé parmi les 39 autochtones de l'étude que dans l'ensemble de la population et que leur dépendance à de telles substances s'échelonnait sur des périodes de 10 à 25 ans. Dubec (1982) signale que la majorité des femmes de l'échantillon était en état d'ébriété au moment de l'arrestation. Si LaPrairie (1984) estime que l'alcool joue un rôle plus important dans les délits pour les femmes autochtones que pour les hommes autochtones, il importe aussi de rendre compte des pratiques des agences de contrôle pénal pour confirmer ou infirmer l'hypothèse d'une tolérance différentielle basée sur le sexe à l'endroit de la consommation d'alcool des autochtones.

$4^{\circ}$ Gravité et insignifiance des criminalisations : les recherches portant sur la nature des incriminations (condamnations et types de délits à l'admission) s'entendent pour relever à la fois l'insignifiance et la gravité des délits enregistrés pour les femmes autochtones par rapport aux autres femmes et même par rapport aux hommes autochtones. Les études portant tant sur les institutions de détention provinciale que fédérale soulignent la plus grande tendance pour les femmes autochtones d'être admises pour des délits de violence contre la personne en comparaison des autres femmes, mais aussi leur tendance à être admises plus régulièrement pour des délits insignifiants par rapport aux allochtones. Dans ce registre de délits, le type d'admissions par excellence des femmes autochtones est le défaut de paiement d'amende (Dubec, 1982; Johnson, 1986; LaPrairie, 1984) et les infractions relatives aux 
règlements municipaux qui comprennent des « délits » tels le désordre, la nuisance, l'ivresse, la flânerie. le tapage, le vagabondage (Jaccoud, 1986). Notre étude sur les admissions des Amérindien-ne-s dans les prisons du Québec de 1977 à 1985 souligne que les Amérindiennes sont deux fois et demie plus souvent admises pour cette dernière catégorie de «délits » que les femmes allochtones et elles le sont près de quatre fois plus souvent que les hommes! (voir tableau 4). L'étude du Centre d'amitié autochtone de Montréal (1986) révèle que la majorité des femmes de l'échantillon avaient été arrêtées sous le motif d'avoir troublé la paix. L'incarcération pour ce type de « délits " est certes révélatrice du statut socio-économique défavorisé de ces femmes (LaPrairie, 1984), mais elle dévoile aussi des pratiques particulièrement répressives à l'endroit de femmes autochtones emprisonnées à répétition pour des vétilles.

$5^{\circ}$ Précarité multi-dimensionnelle : très souvent, les femmes autochtones criminalisées sont arrêtées alors qu'elles sont sans emploi ou bénéficiaires de l'aide sociale. En 1981, le taux de chômage des femmes autochtones incarcérées était 8 fois plus élevé que la moyenne nationale (Johnson, 1986). Leur niveau d'éducation est généralement bas : $\mathbf{5 2}$ pour cent des femmes autochtones interviewées dans l'étude de Dubec (1982) avaient moins qu'une huitième année et 97 pour cent moins qu'une onzième année ; elles ont majoritairement au moins un enfant placé dans des centres d'accueil. Une responsable du programme pour le Native Police Liaison Unit du service de police de Vancouver prétendait d'ailleurs que le premier contact avec le système de justice se faisait souvent par l'intermédiaire du tribunal de la famille au moment où les enfants sont arrachés du foyer (Canada, Solliciteur général, 1986, p. 20).

$6^{\circ}$ Victimisation : les diverses formes de violence et d'abus que subissent les femmes autochtones dans les réserves et communautés nordiques commencent à peine à être mises au grand jour. L'étude sur les femmes purgeant une sentence fédérale (Canada, Groupe d'étude sur les femmes purgeant une peine fédérale, 1990) rapportait que 90 pour cent des autochtones interviewées déclaraient avoir été physiquement abusées, et ce habituellement sur de longues périodes, alors que les femmes allochtones témoignaient du même genre d'abus dans 61 pour cent des cas.

$7^{\circ}$ Isolement socio-culturel : la plupart d'entre elles seraient originaires de réserves qu'elles ont quittées ou fuies pour venir s'installer dans des centres urbains. Par exemple, la population autochtone de 
Montréal, évaluée à quinze mille personnes, serait majoritairement composée de femmes qui ont fui la violence et les abus sexuels dont elles ou leurs enfants ont été la cible. Une intervenante du Centre d'amitié de Québec estime que les deux tiers des femmes autochtones qui viennent s'installer en ville le font pour cette raison (Chaumel, 1991, p. 13). Les conditions de vie en milieu urbain sont alors altérées par les barrières culturelles et linguistiques qui maintiennent les femmes autochtones dans un état d'isolement qui amplifie certainement leur vulnérabilité.

\section{LES ANALYSES DE LA SURREPRÉSENTATION}

Les tentatives d'appréhension et de compréhension du phénomène de la surreprésentation des femmes autochtones sont encore timides. Il faut davantage parler de modèles explicatifs que de productions thériques originales. Ces modèles pèchent par leur tendance au « pénalocentrisme», penchant qui tire son origine de l'accent mis sur la surreprésentation. Désormais, c'est la notion de surreprésentation qui est «surreprésentée », et ce méta-objet tend à contenir toute velléité théorique. Les analyses plongent dans une arithmétique déracinée de tout contexte socio-historique (voir par exemple l'étude de Hagan, 1986) et font rarement la distinction selon le sexe. Nous présentons un éventail des principales thèses avancées pour « expliquer » les démêlés des autochtones avec le contrôle pénal, en précisant que ces modeles ont été produits sans égard au sexe mais qu'ils impliquent la femme autochtone criminalisée.

\section{$1^{\text {o }} \quad$ Les conditions socio-économiques}

L'explication par les conditions socio-économiques représente l'un des modèles explicatifs classiques et peut prendre deux trajectoires théoriques opposées. Dans une perspective positiviste, les conditions socio-économiques viennent expliquer le passage a l'acte. La femme autochtone appartiendrait alors à cette culture du pauvre caractérisée par des comportements déviants et criminels. Dans une perspective plus critique, ces mêmes conditions socio-économiques vont expliquer la réaction sociale à l'endroit des femmes autochtones, soit en insistant sur la stigmatisation de leur marginalité socio-économique (dans ce cas, l'accent est mis sur les relations entre la police et les groupes ethno-culturels (Griffiths et Yerbury, 1984) ou sur un traitement differentiel plus sévère des tribunaux (Hartnagel, 1975)), soit en soulignant l'importance 
des conditions socio-économiques qui influent sur la vulnérabilité des autochtones, plus démunis et donc pénalisés par le système de justice. LaPrairie (1990) suggère que la prise en compte de facteurs tels que l'emploi ou la présence de soutiens familiaux et communautaires dans l'octroi des sentences ou des libérations conditionnelles exerce une forte discrimination à l'encontre des représentantes des premières nations issues de communautés socio-économiquement défavorisées.

\section{$2^{\circ} \quad$ L'alcool $^{\prime}$}

Toutes les études portant sur les autochtones et leurs démêlés avec la justice font état de l'incidence de l'alcool. Plusieurs thèses peuvent être retracées. La thèse classique du rôle criminogène de l'alcool établit un lien direct ou indirect entre l'alcool et les infractions commises par les autochtones (Finkler, 1980 ; Schmeiser, 1974). La thèse socio-économique de Harding suggère des rapports entre le chômage, les problèmes familiaux et l'ivresse dans les lieux publics et soutient que le système de justice pénale est utilisé pour combler un vide dans les programmes sociaux et économiques. Selon ce modèle d'analyse, l'insuffisance ou le manque de programmes sociaux et économiques pour les clochards crée des pressions sur les forces policières et les ivrognes se serviraient du système de justice (prison) comme abri (in Haveman $e t$ al., 1984, p. 148). Suivant la thèse du sous-développement, la consommation d'alcool est envisagée comme un moyen facilement accessible d'affronter les frustrations engendrées par les conséquences du sous-développement que connaissent les nations autochtones (Harding, 1978). La thèse de la visibilité sociale précise que l'enivrement dans les lieux publics augmente le risque d'arrestation (Hawthorn et al., 1975) alors que la thèse de la visibilité culturelle soutient que les habitudes de consommation de la femme autochtone (tout comme celles de l'homme) sont différentes de celles des allochtones. L'étude de Shkilnyk (1985) suggère un pattern différentiel face à l'alcool : boire serait une activité sociale et les autochtones n'éprouveraient pas la même dépendance physiologique ; ils peuvent par exemple s'abstenir de boire dans l'attente du prochain chèque d'aide sociale. mais quand ils boivent, ils consomment jusqu'à perdre conscience. Par conséquent, les comportements seront plus visibles dans la mesure où ils s'insèrent dans une pratique collective très autodestructrice. Enfin, nous pourrions suggérer une thèse de la discrimination législative qui a contribué à rendre visibles et à contrôler les comportements des autochtones par la production de lois discriminatoires telles que la Loi sur les Indiens de 1876 dont certaines dispositions interdisaient la consommation d'alcool dans les 
réserves, obligeant l'autochtone à sortir de sa réserve pour consommer légalement des boissons alcooliques.

Ces thèses permettent de comprendre certains aspects des liens qui unissent les autochtones au système de justice pénale, mais elles ne permettent certainement pas d'en comprendre la dynamique générale. Verdun-Jones et Muirhead (1979-1980) déplorent d'ailleurs le manque d'explications théoriques sur le rapport entre l'alcool et le taux de criminalité chez les autochtones. Il est aussi primordial de se pencher sur l'analyse du traitement que réserve la police aux femmes autochtones dans les centres urbains en rapport à leurs habitudes de consommation d'alcool.

\section{$3^{\circ}$ Les facteurs démographiques}

LaPrairie (1990) prévient qu'il faut se garder de conclure trop hâtivement à la surreprésentation des autochtones dans le système de justice. La notion de surreprésentation renvoie au pourcentage d'autochtones dans les institutions correctionnelles comparativement à leur pourcentage dans la population générale. L'introduction de la variable âge dans la comparaison des populations autochtones et non-autochtones permet de se rendre compte que la tranche d'âge des 14-25 ans est nettement plus forte chez les premières nations en raison des taux de natalité plus élevés et d'une espérance de vie moindre. Or il s'agit du groupe d'âge pour lequel le risque d'incarcération est le plus élevé. L'âge serait par conséquent un facteur démographique évolutif qui permettrait non seulement de comprendre la surreprésentation des hommes et des femmes autochtones, mais encore de la nuancer.

\section{$4^{\circ} \quad$ La visibilité}

Cette thèse suggère que la surreprésentation des femmes autochtones dans le système pénal soit attribué à des modèles de crimes différents comparativement aux non-autochtones. En particulier, les femmes autochtones tendraient à être impliquées dans des modèles de crimes plus graves que les allochtones (LaPrairie, 1989a). Aussi la surreprésentation s'expliquerait-elle par l'implication dans un mode délictuel plus repérable par les agents de contrôle puisque basé sur la visibilité et la gravité des comportements (LaPrairie, 1990). Visibilité ne rime pas qu'avec gravité, puisque nous avons souligné que l'incarcération pour des accrocs mineurs à l'ordre public (tapage, vagabondage) était chose courante pour les femmes autochtones (Jaccoud, 1986). Corollairement, des études comparatives ont montré que les taux de réso- 
lution étaient nettement plus importants dans les communautés autochtones que chez les non-autochtones et que la visibilité des comportements était renforcée par des taux d'encadrement policier plus élevés (Hyde et LaPrairie, 1987). Si la taille des communautés autochtones est une donnée importante pour comprendre la " sur-visibilité » des événements susceptibles d'entrer dans la filière pénale, elle est une donnée sans doute moins pertinente dans le cas des femmes qui semblent entrer en contact avec l'appareil pénal principalement en contexte urbain.

\section{$5^{\circ} \quad$ Les différences culturelles}

De nombreux travaux font état de la vulnérabilité des autochtones devant un système de justice qui leur est culturellement étranger. L'incompréhension généralisée et générale du système juridique euro-canadien, la non-adhésion aux valeurs et à la philosophie de l'administration de la justice seraient des facteurs susceptibles d'affaiblir les capacités de défense et de résistance des autochtones. Par exemple, la tendance des autochtones à plaider coupable dans notre système de droit est souvent rapportée comme un facteur contribuant à leur incarcération excessive. Or, l'amendement par la reconnaissance publique de sa faute est entre autres l'un des mécanismes traditionnels de résolution des conflits dans les sociétés communautaires (Rouland, 1979 et 1983).

\section{$6^{\circ} \quad$ La discrimination policière}

Certaines études font état d'une discrimination policière à l'endroit des autochtones alimentée par des préjugés raciaux (Griffiths et Yerbury, 1984 ; Bienvenue et Latif, 1974). Sans que cette thèse soit à rejeter, il faut admettre que les données sur les pratiques policières sont méthodologiquement pleines d'embûches. Car conclure à des pratiques discrétionnaires négatives à l'endroit des autochtones suppose l'application de méthodes d'observation participante difficilement réalisables (LaPrairie, 1990). Mais des études orientées vers les représentations des policiers allochtones, et même autochtones, permettraient tout de même de mettre au jour des données sur les préjugés éventuels des forces de l'ordre vis-à-vis les premières nations, et tout particulièrement à l'endroit des femmes.

\section{$7^{\circ} \quad$ Le traitement différentiel}

L'hypothèse qui veut qu'un traitement différentiel explique la surreprésentation des autochtones est sans doute l'une des hypothèses les plus souvent mises de l'avant. Elle chapeaute trois orientations possibles. La première soutient que les autochtones font l'objet d'un traite- 
ment sentenciel plus sévère de la part des juges (Hartnagel, 1975) ; la seconde croit que ce n'est pas un traitement différentiel qui rend compte de la surreprésentation des autochtones, mais l'application égalitaire du droit qui se répercute négativement sur les autochtones défavorisés par rapport aux autres Canadiens (Hagan, 1977). Les femmes autochtones, défavorisées par rapport aux autres femmes, ne feraient donc pas l'objet d'une discrimination sentencielle mais subiraient les contre-coups des inégalités structurelles vécues dans la société eurocanadienne. L'emprisonnement pour défaut de paiement d'amendes, plus courant chez les femmes autochtones que chez les non-autochtones (Johnson, 1986 ; LaPrairie, 1989a), corroborerait la thèse de Hagan. La dernière considère que les autochtones reçoivent un traitement sentenciel preférentiel. Ainsi, les sentences d'emprisonnement des femmes autochtones seraient moins longues que celles des non-autochtones (Clark, 1989; Johnson, 1986). Ces pratiques pourraient être adoptées consciemment par les juges pour contrebalancer les effets négatifs des disparités socio-économiques (Archibald in LaPrairie, 1990).

Ces différentes thèses ont surtout été produites pour tenter d'expliquer la fréquence des démêlés des autochtones avec le système de justice pénale, sans distinction de sexe. Or, nous l'avons souligné, la surreprésentation des femmes autochtones est proportionnellement encore plus accentuée que celle des hommes et cette situation réclame une attention particulière qui a peine à émerger. La théorie sociostructurelle de LaPrairie (1987) est à ce jour la seule contribution théorique qui intègre une réflexion en fonction du sexe pour comprendre la fréquence des démêlés des autochtones avec l'appareil pénal.

\section{LA THÉORIE SOCIO-STRUCTURELLE DE LAPRAIRIE}

LaPrairie propose un modèle pour comprendre la surcriminalisation des femmes autochtones au Canada qui s'inscrit dans la tradition anthropologique des théories colonialistes et du sous-développement traduisant les rapports de l'État avec les autochtones (Kellough, 1980; Frideres, 1974 ; Patterson, 1972).

L'originalité de ce modèle est qu'il tient compte de la double insertion sociale de la femme autochtone, tant dans la structure sociale au Canada qu'à l'intérieur des communautés autochtones. S'inspirant des travaux de Davis (1971) et de Kellough (1980), LaPrairie estime que la société canadienne est doublement hiérarchisée. Sur le plan interne, le Canada est un pays de colonisation dans lequel des terres ont 
été colonisées par des métropoles domestiques ou internes comme Toronto ou Montréal. Ces terres, selon la tradition des théories du sousdéveloppement et de la dépendance, constituent des périphéries socioéconomiquement dépendantes de centres dominants. Sur le plan externe, et en référence au système économique mondial, ce sont les métropoles domestiques qui deviennent dépendantes de métropoles étrangères. Les rapports entre centres et périphéries s'instaurent selon une structure qui s'emboîte : chaque centre a sa périphérie et la périphérie devient le centre d'une autre périphérie et ainsi de suite. Par exemple, Val-d'Or est périphérique à Montréal, Montréal étant elle-même une périphérie par rapport à New York, mais Val-d'Or constitue le centre d'une périphérie comme Povungnituk, communauté nordique inuit du Nouveau-Québec. En tenant compte du niveau interne et externe de structuration du système social, on voit que centre et périphérie peuvent voir leur statut alterner. Or, les communautés autochtones se situent à l'extrême limite de cette structuration et n'occupent qu'un statut de périphérie. C'est là une des caractéristiques des régions sousdéveloppées et les communautés indiennes sont passées, à travers le processus de colonisation interne, d'un statut de «territoires fortement productifs » à un statut de «territoires productifs marginaux ». Aujourd'hui, selon l'auteure, les économies locales indiennes sont «sousdéveloppées", et ce sous-développement résulte d'une politique de subordination économique délibérée. Si les communautés autochtones ont pu jusqu'au milieu du $\mathrm{XX}^{\mathfrak{e}}$ siècle préserver une économie de subsistance à travers les activités de chasse, de pêche, de trappe et d'agriculture, cette économie a succombé à la politique de l'État-providence des années 1950. Et pour LaPrairie, la politique de domination de l'État a eu pour effet de déstructurer ces communautés à un double niveau : a) les rapports entre hommes et femmes et b) les rapports entre générations. L'économie traditionnelle autochtone reposait sur l'égalitarisme et la coopération. Les rôles, quoique distincts, n'en étaient pas moins assumés par les temmes et les hommes sans dévalorisation et hiérarchisation. La transformation de l'économie traditionnelle, sous l'impact de la société euro-canadienne, serait alors venue bouleverser les rapports entre hommes et femmes en transformant les rôles de chacun. Le système éducatif, imposé aux autochtones. a également contribué à détériorer les relations au sein de la famille puisqu'il a amorcé une coupure entre les générations en inculquant des valeurs différentes aux enfants autochtones retirés de leurs communautés pour être placés dans des pensionnats. LaPrairie estime que les hommes ont perdu davantage que les femmes à travers le processus de colonisation ; la femme a réussi à conserver certaines activités traditionnelles alors que l'homme aurait 
pratiquement tout perdu en perdant son rôle de pourvoyeur de la communauté et de la famille. Les activités traditionnelles de chasse et de pêche ayant perdu leur sens, les femmes auraient pris la relève en devenant les pourvoyeurs de la famille. C'est là la thèse centrale de LaPrairie pour expliquer la détérioration des relations entre les sexes et comprendre en retour les agressions et les violences subies par les femmes devenues les victimes des frustrations accumulees par les hommes. La logique du modele s'éclaire: les frustrations vécues à travers le processus de dépendance, de dépossession, de transformations et de pertes engendrent de l'agressivité, agressivité amplifiée par l'abus de drogues et d'alcool. Le besoin de pouvoir ne s'exercerait plus alors qu'à travers des comportements de violence :

The satisfaction of power needs, denied in the roles made available to Indians in the larger society, may be found in exercising control over vulnerable others. Thus, rape, child sexual abuse, and wife battering are being reported with increasing frequency in Indian communities in recent years. (LaPrairie, 1987, p. 108.)

Et c'est à ce stade de la démonstration que le modèle de LaPrairie trahit quelques faiblesses. Car nous glissons dans un modèle explicatif du comportement traditionnel qui analyse l'impact de la colonisation et du sous-développement des communautés autochtones sur le comportement criminel des femmes autochtones (LaPrairie, 1989a, p. 89). LaPrairie émet alors l'hypothèse d'une relation causale entre la violence des hommes sur les femmes et les activités criminelles des femmes. Sa construction logique est alors que :

$1^{\circ}$ les comportements de violence des femmes autochtones seraient liés aux victimisations subies ;

$2^{\circ}$ leur migration dans les centres urbains serait conditionnée par le désir d'échapper aux victimisations vécues dans les réserves. Le phénomène de la migration résulte aussi des conséquences des dispositions sexistes de la Loi sur les Indiens qui, avant leur amendement en 1985 , éteignaient le statut des Amérindiennes qui épousaient un non-Indien. Cette législation est importante pour comprendre la migration des Amérindiennes dans les villes et les conséquences psycho-sociologiques d'une telle perte d'identité. Mais les études n'apportent encore aucune donnée permettant d'articuler la discrimination sexuelle de la Loi sur les Indiens avec la surpénalisation des femmes autochtones : 
$3^{\circ}$ les femmes autochtones subiraient dans les villes une discrimination combinée à un faible niveau d'éducation qui les relégueraient alors à des situations de non-emploi ;

$4^{\circ}$ les difficultés éprouvées dans leur vie urbaine faciliteraient le recours aux drogues et à l'alcool et aux activités de prostitution, pratiques qui accroissent leur chance d'un rapport conflictuel avec la loi. Et le simple fait de se retrouver dans une ville augmenterait, selon LaPrairie, leur visibilité face à la police. Là encore, il faut reconnaître que l'état précaire des données ne permet pas clairement d'aboutir à de telles conclusions.

La théorie socio-structurelle de LaPrairie a le mérite d'élaborer un modèle qui tienne compte à la fois de facteurs socio-historiques et de facteurs psycho-sociaux. Il est regrettable que l'auteure, qui construit son modèle en s'inspirant des théories de la dépendance et du colonialisme, ne suive pas sa logique. Alors qu'elle conçoit le système économique et le système éducatif euro-canadiens comme des instruments ayant concouru à la domination, le système de justice est isolé et n'est plus envisagé que comme une instance réagissant à un comportement produit par des instruments de colonisation extérieurs à lui. Le modèle de LaPrairie aurait tout à gagner dans la compréhension des rapports qu'entretiennent l'appareil de justice pénale et les femmes autochtones s'il amorçait une réflexion sur la place et le rôle du système de justice dans l'œuvre d'assimilisation des peuples autochtones. Ce sont d'ailleurs les femmes autochtones elles-mêmes qui amorcent cette analyse en considérant le système de justice comme un prolongement de leur institutionnalisation et de leur mise sous tutelle historique (Canada, Groupe d'étude sur les femmes purgeant une peine fédérale, 1990 ; Faith et al., 1990: Sugar et Fox, 1989-1990). Monture (1989) avertit d'ailleurs que la surreprésentation des autochtones s'étend à l'ensemble des institutions d'enfermement. Par exemple, en 1977, la population des enfants autochtones placés en foyer d'accueil était estimée à quinze mille enfants, soit 20 pour cent des enfants placés (p. 2). 11 faut donc s'intéresser de près à l'institutionnalisation des femmes autochtones dans son ensemble si l'on veut comprendre un tant soit peu les liens étroits qui les unissent au système pénal.

\section{CONCLUSION}

Cet article a voulu faire le point sur les connaissances acquises dans la problématique des femmes autochtones et du système pénal. 
Bilan sommaire pour des acquis encore fragiles! Les données se construisent peu à peu, tant et si bien qu'une pluie de chiffres s'est abattue sur les nations autochtones du Canada: des taux de suicides, de chômage, d'arrestations, d'incarcérations et de victimisations plus criants que pour le reste de la population. Mais cette averse semble avoir noyé l'imagination des chercheurs qui jusqu'ici n'ont pas fait preuve d'une création théorique débordante. Prise entre des statistiques et l'acuité politique de la « question autochtone », la recherche démarre, hésite et s'essouffle déjà. La redondance est devenue coutume pour un objet pourtant jeune. En 1970, la recherche đébutait sur l'incarcération excessive des hommes et des femmes autochtones. Vingt ans plus tard, c'est de surreprésentation qu'il est question. Les études s'engluent dans une réalité figée. Une réalité largement habitée par les femmes autochtones qui demeurent pourtant les grandes oubliées de la recherche et des politiques. Les regroupements de femmes autochtones ont su déclencher l'alarme et il faut souhaiter que cet éveil se poursuive. Mais force est d'admettre que l'analyse dans le domaine de la justice pénale et des femmes autochtones est quelque peu étouffée par le contexte politique des revendications territoriales et autonomistes des premières nations. Elle est prise entre l'urgence de l'action (LaPrairie, 1989b) et l'émotivité des débats. Sans pour autant prôner la ségrégation, il serait utile de démarquer le champ de l'action du champ théorique pour mener le travail d'analyse et de réflexion qui s'impose. Dans le champ de l'action, il est urgent de dépasser le stade de la dénonciation pour véritablement passer à une politique qui satisfasse les revendications des femmes autochtones. La difficulté majeure est moins d'être à l'écoute des voix qui s'élèvent que d'orienter une politique cohérente. Les autochtones eux-mêmes sont divisés entre l'option de l'autochtonisation du système de justice euro-canadien et celle de la séparation. Dans le champ théorique, il est grand temps de penser les femmes autochtones dans leur relation au pénal en travaillant l'articulation criminalisation et colonisation tout en sortant du positivisme aveugle qui a caractérisé les études sur les femmes autochtones, sans pour autant tomber dans un constructivisme étroit. Les femmes autochtones sont en proie à des difficultés que l'on ne peut nier mais qu'il faut comprendre comme l'une des conséquences du joug qu'elles ont subi à travers le processus de colonisation et d'acculturation. Et c'est dans ce complexe qu'il s'agit de dégager le rôle du système pénal dans ce processus pour mettre à jour les pratiques de ses agents à leur égard. 


\section{BIBLIOGRAPHIE}

BIENVENUE, P. M. ; LATIF, A. H. (1974), " Arrests, Dispositions and Recidivism : A Comparison of Indians and Whites $\#$, Revue canadienne de criminologie, vol, 16, $\mathrm{n}^{\circ} 2$, pp. 105-116.

CANADA. GROUPE D'ÉTUDE SUR LES FEMMES PURGEANT UNE PEINE FÉDÉRALE (1990), La Création de choix, s. p.

CANADA. SOLLICITEUR GÉNERAL (1989), Rapport final du groupe d'étude sur les autochtones au sein du régime correctionnel fédéral, Ministre des Approvisionnements et Services Canada.

CANADA. SOLLICITEUR GÉNÉRAL (1986), « Les fernmes ayant des démêlés avec la justice : hier et demain ${ }^{2}$, Liaison, vol. $12, n^{\circ} 9$, pp. 18-23, Ottawa, Division des communications, Direction des programmes.

CENTRE D'AMITIE AUTOCHTONE DE MONTRÉAL (1986), Native Women in Conflict with the Law, s. p.

CHAUMEL, G. (1991), « Vivre en ville, pas vraiment une solution ", Rencontre, vol. $12, \mathrm{n}^{\circ} 4$, pp. 12-13, Gouvernement du Québec, Secrétariat aux affaires autochtones.

CLARK, S. (1989), Sentencing Patterns and Sentencing Options Relating to Aboriginal Offenders, Ottawa, Department of Justice, Canada.

COUSINEAU, M.-M., LABERGE, D. et THEORET, B. (1986), Prisons et prisonniers : une analyse de la détention provinciale québécoise durant la dernière décennie, rapport de recherche, Montréal, Universitê du Québec à Montréal, Département de sociologie.

DAVIS, A. K. (1971), * Canadian Society and History as Hinterland Versus Metropolis $»$, R. Ossenberg (éd.) Canadian Society : Pluralism, Change, and Conflict, Scarborough, Ontario, Prentice-Hall, pp. 6-32.

DUBEC, B. (1982), Native Wonten and the Criminal Justice System : An Increasing Minority, Thunder Bay, Ontario Native Women's Association.

FAITH, K. et al. (1990), « Native Women in Canada : A Quest for Justice », Social Justice, vol. 17, $\mathrm{n}^{\circ} 3$, pp. 167-188.

FAULKNER, C. (1989), Etude sur les délinquants inuit, Service correctionnel du Canada, Ministère du Solliciteur général.

FINKLER, H. (1980), Les Inuit et l'administration de la justice. Le cas de Frobisher Bay (T. N. O. ), Hurtubise HMH.

FRIDERES, J. S. (1974), Canada's Indians : Contemporary Conflicts, Toronto, Prentice-Hall of Canada.

GRIFFITHS, C. T. et YERBURY, J. C. (1984), « Natives and Criminal Justice Policy : The Case of Native Policing *, Revue canadienne de criminologie. vol. $26, \mathrm{n}^{\circ} 2$, pp. 147-160. 
HAGAN, J. (1986), * Toward A Structural Theory of Crime, Race and Gender », R. H. Silverman (éd.) Crime in Canadian Society, pp. 189-201, $3^{\mathrm{e}}$ édition, Toronto, Vancouver, Butterworths.

HAGAN, J. (1977), « Finding Discrimination: A Question of Meaning », Ethnicity, vol. 4 , pp. 177-187.

HARDING, J. (1978), « Development, Underdevelopment and Alcohol Disabilities in Northern Saskatchewan », Altematives, vol. $7, \mathrm{n}^{\circ} 4, \mathrm{p} .30$.

HARTNAGEL, T. H. et WYNNE, D. F. (1975), « Plea Negociation in Canada », Revue canadienne de criminologie, vol. $17, \mathrm{n}^{\circ} 1, \mathrm{pp} .45-56$.

HAVEMANN, P. et al. (1984), La Loi et les Autochtones au Canada, Rapport pour spécialistes n ${ }^{\circ}$ 1984-7, Ministère du Solliciteur général du Canada, Ottawa, Information Canada.

HAWTHORN, H. B., BELSHAW, C. S. et JAMIESON, S. M. (1975), « The Indians of British Columbia and Alcohol $»$, Alcoholism Review, vol. 2, n 3, pp. 10-14.

HYDE, M. et LAPRAIRIE, C. (1987), Amerindian Police Crime Prevention, Ottawa, Ministère du Solliciteur Général, $n^{\circ}$ 1987-21.

JACCOUD, M. (1986), Les Autochtones dans le système de justice pénale au Canada et au Québec, thèse de maîtrise, École de criminologie, Université de Montréal, inédit.

JOHNSON, H. (1986), Les Femmes et la criminalité au Canada, Direction des programmes, Secrélariat du ministère, Solliciteur général.

KELLOUGH, B. (1980), "From Colonialism to Imperialism: The Experience of the Canadian Indians ", J. Harp et J. R. Hofley (ed.), Structured lnequality in Canada, Scarborough, Ont., Prentice Hall, pp. 343-377.

LAING, A. (1967), Indians and the Law, Ottawa, Queen`s Printer.

LAPRAIRIE, C. (1990), " The Role of Sentencing in the Over-Representation of Aboriginal Pcople in Correctional Institutions ", Revue canadienne de criminologie, vol. 32, $\mathrm{n}^{\circ} 3$, pp. 429-440.

LAPRAIRIE, C. (1989a), « Some lssues in Aboriginal Justice Research : The Case of Aboriginal Women in Canada $"$, Women and Criminal Justice, vol. $1, \mathrm{n}^{\circ} 1, \mathrm{pp} .81$ 92.

LAPRAIRIE, C. (1989b), « La justice pénale chez les autochtones du Canada. Principes et pratiques ", Anthropologie et Sociétés, vol. 13, n" 1, pp. 143-154.

LAPRAIRIE, C.. ADELBERG, E. et CURRIE, C. (cd.) (1987), “ Native Women and Crime in Canada : A Theoretical Model ", Too few to count. Canadian Wonten in Conflict with the Law, Press Gang Publishers, Vancouver, pp. 103-112.

LAPRAIRIE, C. (1984), "Sclected Criminal Justice and Socio-Demographic Data on Native Women ", Revue canadienne de criminologie, vol. 26, $\mathrm{n}^{\circ}$ 2, pp. 147-160.

MONTURE, P. (1989), «A Vicious Circle: Child Welfare and the First Nations », Canadian Journal of Women and the Law. vol. 3, $\mathrm{n}^{\circ} 1, \mathrm{pp} .1-17$. 
PATTERSON, E. P. (1972), The Canadian Indian. A History since 1500, CollierMacmillan.

ROULAND, N. (1983), "L'acculturation judiciaire chez les Inuit au Canada », Recherches amérindiennes au Québec, vol. XIII, $\mathrm{n}^{\circ} 3$, pp. 179-191, et vol. XIII, $\mathrm{n}^{\circ} 4$, pp. 307-318.

ROULAND, N. (1979), "Les modes juridiques de solution des conflits chez les Inuit ", Études/Inuit/Studies, vol. 3, numéro hors-série, $171 \mathrm{p}$.

SCHMEISER, D. H. (1974), Délinquance chez les autochtones et la loi, Commission de réforme du droit du Canada. Ottawa.

SECRETARIAT DES ACTIVITÉS GOUVERNEMENTALES EN MILIEU AMÉRINDIEN ET INUIT (SAGMAI) (1984), Nations autochtones du Québec, Gouvernement du Québec.

SERVICE CORRECTIONNEL CANADA (1985), Rapport du profil de la population féminine. Population au registre $12 / 31 / 1984$.

SHKILNYK, A. (1985), A Poison Stronger than Love: The Destruction of an Ojibwa community, New Haven, Yale University Press.

STATISTIQUE CANADA (1991), « Les contrevenantes adultes dans les systèmes correctionnels provinciaux et territoriaux, 1989-1990 ", Juristat, vol. 11, $\mathrm{n}^{\circ} 6$, pp. 1-10, Catalogue 85-002.

STATISTIQUE CANADA (1989), « Caractéristiques de la population et des logements. Origine ethnique et citoyenneté ", Le Pays, Canada 1986, Catalogue 93109, Ottawa, Ministre des Approvisionnements et Services Canada.

SUGAR, F. et FOX, L. (1989-1990), « Nitsum Peyako Seht' wawin Iskwewak : Breaking the Chains $»$, Canadian Joumal of Women and the Law, vol. $3, \mathrm{n}^{\circ} 2$, pp. 465 482.

VERDUN-JONES S. et MUIRHEAD, G. (1979-1980), "Natives in the Criminal Justice System : An Overview ", Crime and/et Justice, vol. 1, $\mathrm{n}^{\mathrm{os}}$ 7-8, pp. 3-21. 